\title{
Microinvasion: could it be sufficient diagnostic criteria for the optimal treatment decision?
}

\author{
Darko Zdravkovic $^{1,2} \odot$. Nebojsa Ivanovic ${ }^{1,2} \cdot$ Bogdan Crnokrak $^{1}$
}

Received: 17 April 2019 / Accepted: 3 May 2019 / Published online: 9 May 2019

(c) Springer Science+Business Media, LLC, part of Springer Nature 2019

\section{Introduction}

\section{Letter to the Editor}

We have read with great interest the article by HolmRasmussen et al. that evaluated the incidence and risk factors of sentinel lymph node (SN) and non-SN metastases in patients with microinvasive breast cancer [1]. In this very comprehensive and interesting article, the authors suggest omission of axillary staging in microinvasive breast cancer patients. Regarding this very important issue, we would like to highlight our point of view.

It is well known that microinvasion is a condition related with ductal carcinoma in situ (DCIS). However, the pathogenesis of lymph node metastasis in DCIS and the clinical management of node-positive DCIS still remain controversial. Some authors have described a prevalence of even $58.3 \%$ of microinvasion by histological re-examination of tissue specimens in patients affected by DCIS with concomitant nodal metastasis. Hence, nodal involvement in DCIS depends on the misdetection of occult microinvasion especially in the case of very wide intraductal carcinoma [2].

Undoubtedly, microinvasion is the information from the pathological report and we should have it in mind that optimal treatment decision should be done earlier.

We think that treatment decision regarding regional lymph nodes should be made considering numerous factors such as palpability, tumor size, extension of the mammographic appearance, multicentricity, high-grade lesion, molecular subtype, young age and the type of further surgery. According to our opinion microinvasion should be considered as a single piece of complex mosaic with many

Darko Zdravkovic

drdarkozdravkovic@gmail.com

1 University Medical Center, Bezanijska Kosa, Belgrade, Serbia

2 Faculty of Medicine, University of Belgrade, Bezanijska Kosa Street BB, Belgrade 11080, Serbia information regarding minucious diagnostic and treatment pathways of DCIS patients.

Since the large number of patients may have occult invasive cancer with true metastasis, tumors with occult microinvasion should be treated as node-positive small invasive cancers. Regarding this, we would like to point out that without exhaustive primary tumor sectioning, it is quite difficult to predict the pathogenesis [3].

\section{Compliance with ethical standards}

Conflict of interest The authors declare that they have no conflict of interest.

Ethical approval This article complies with the ethical rules applicable for this journal.

Research involving human and animal participants This article does not contain any studies with human participants or animals performed by any of the authors.

\section{References}

1. Holm-Rasmussen EV, Jensen MB, Balslev E, Kroman N, Tvedskov TF (2019) Sentinel and non-sentinel lymph node metastases in patients with microinvasive breast cancer: a nationwide study. Breast Cancer Res Treat. https://doi.org/10.1007/s10549-01905200-4

2. Bertozzi S, Cedolini C, Londero AP, Baita B, Giacomuzzi F, Capobianco D, Tortelli M, Uzzau A, Mariuzzi L, Risaliti A (2019) Sentinel lymph node biopsy in patients affected by breast ductal carcinoma in situ with and without microinvasion: retrospective observational study. Medicine (Baltimore) 98(1):e13831

3. Yonekura R, Osako T, Iwase T, Ogiya A, Ueno T, Kitagawa M, Ohno S, Akiyama F (2019) Prognostic impact and possible pathogenesis of lymph node metastasis in ductal carcinoma in situ of the breast. Breast Cancer Res Treat 174(1):103-111

Publisher's Note Springer Nature remains neutral with regard to jurisdictional claims in published maps and institutional affiliations. 